\title{
CREATING VIRTUAL FIELD TRIPS USING 3D MODELS, 3D MAPS, GIGAPIXEL IMAGES, AND 360-DEGREE PANORAMAS IN SAGUACHE COUNTY, COLORADO
}

\section{ABSTRACT}

We have created 3D maps with 3D tiling capability, 3D rock \& fossil models, and 360degree aerial panoramas (AP360) for Virtual Field Trips (VFTs) in Saguache County, Colorado. We have also created 2D tiled gigapixel images in Kingsland, Texas. These VFT tools will help students better learn the geographical and geological importance in those areas. By utilizing various $2 \mathrm{D}$ and $3 \mathrm{D}$ VFT tools with lots of geospatial data, students will be able to view the geology outcrops in a variety of ways leading to multidimensional learning. It will also help students improve their sense of place by using geographical relationship and the geospatial data.

\section{INTRODUCTION}

We have created four VFT tools to organize and integrate the student learning environments that will be used in a traditional geology field camp. The four VFT tools are summarized below:

- Gigapixel images with tiling capability for quick loading in spite of their huge format.

- High definition 3D rock and fossil models reconstructed by Structure from Motion (SfM) and Multi View Stereo (MVS) Photogrammetry.

- Virtual tour web applications using 360degree aerial panoramas with a variety of information such as PDF documents, images, videos, web links, and 3D models.

- 3D Map Platform with tiling capability. Sputnik Web and Cesium ION are webbased 3D map platforms where users can embed their geodata such as sets of aerial photographs, a single georeferenced image, 3d models, and digital terrain models (DTMs). A few of supported file formats for Sputnik Web are CZML, KML, KMZ, SHP, and TLS.

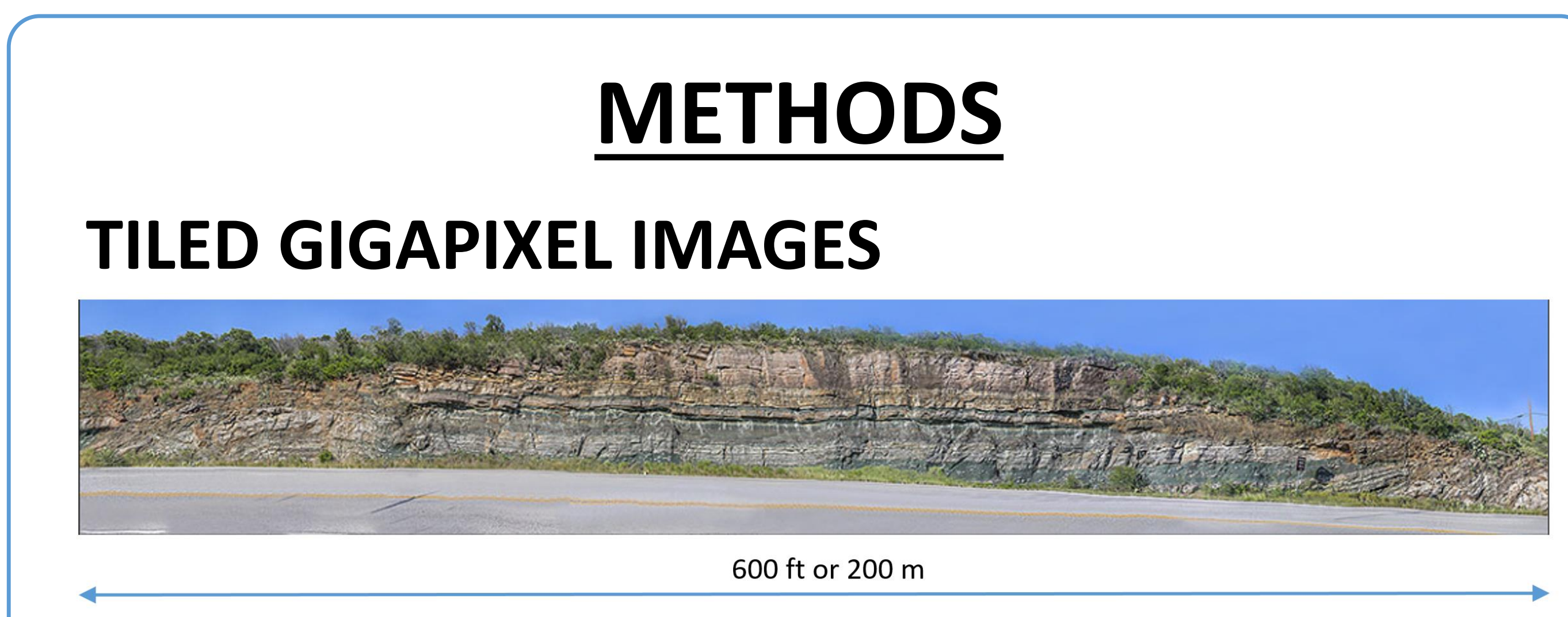

We have created 2D tiled images that provide the entire view and the fine details at the same time. Tiling technologies helped the image be quickly be loaded in spite of its huge dimension $(45,111$ $x 5,684$ pixels)

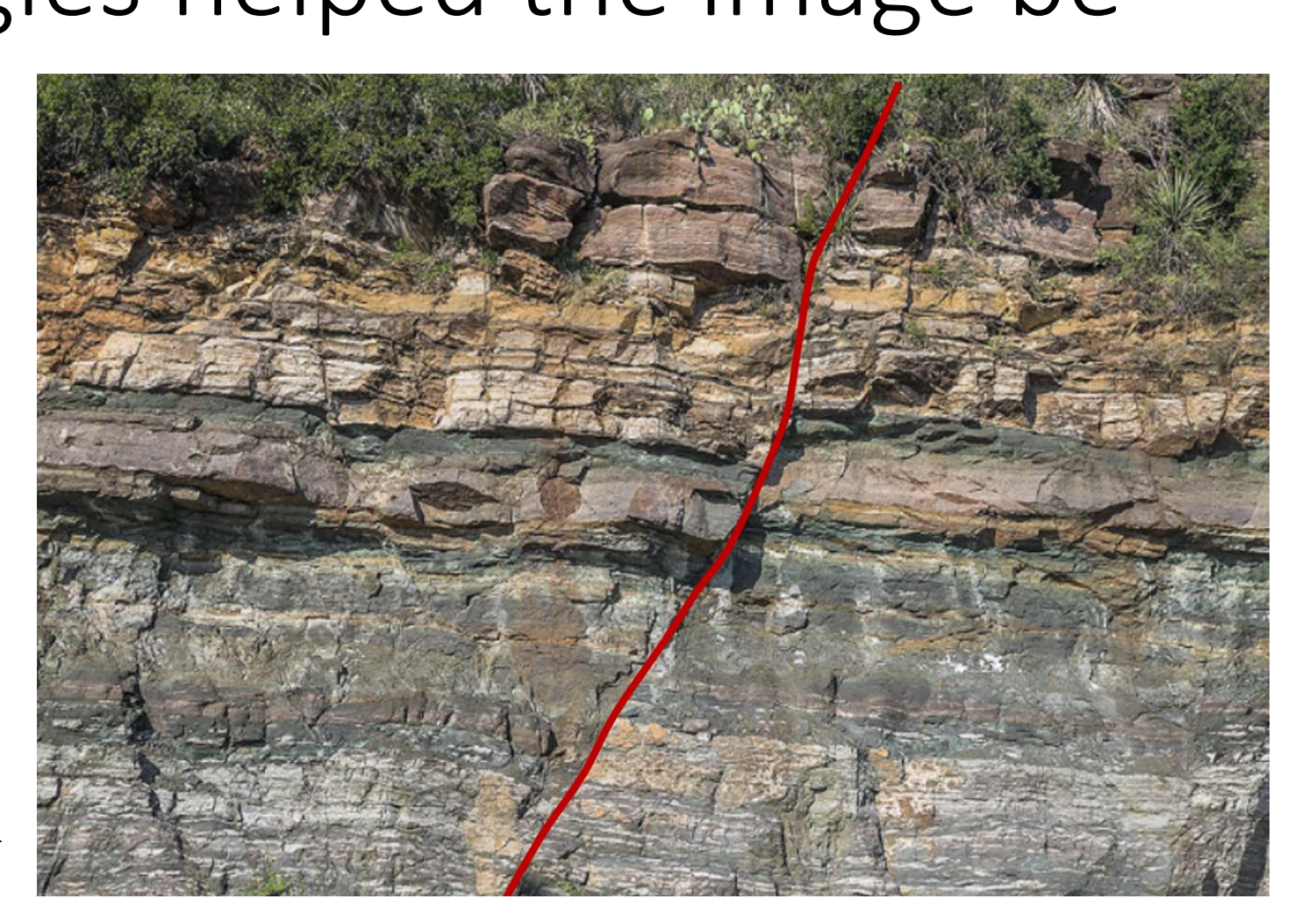

3D ROCK AND FOSSIL MODELS

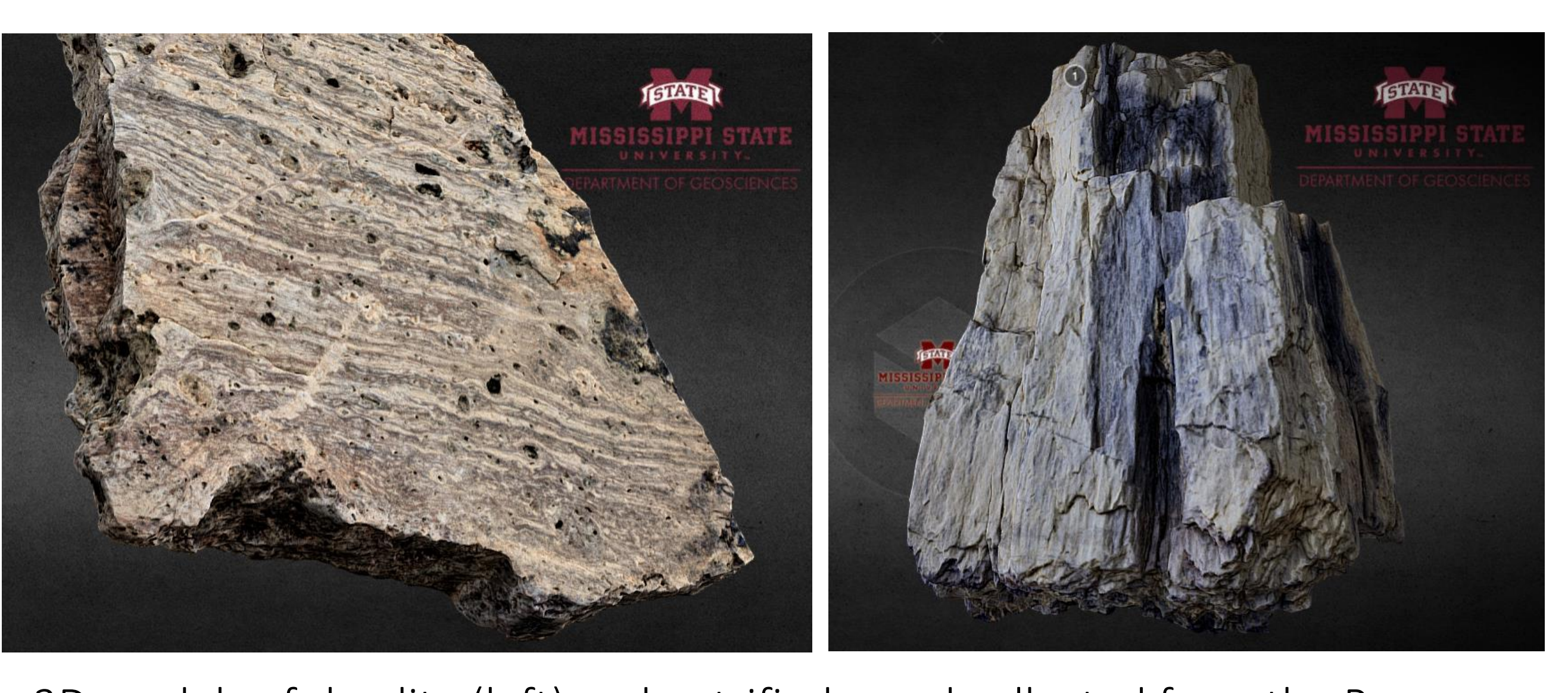

3D models of rhyolite (left) and petrified wo
Rock and Table Top, Saguache County, CO.

We have created 3D models of rocks and fossils using SfM-MVS photogrammetry. The quality of 3D models has greatly improved by enhanced photographic techniques.

VIRTUAL TOUR USING 360-DEGREE AERIAL PANORAMAS AND A VARIETY OF INFORMATION EMBEDDED IN SCENES

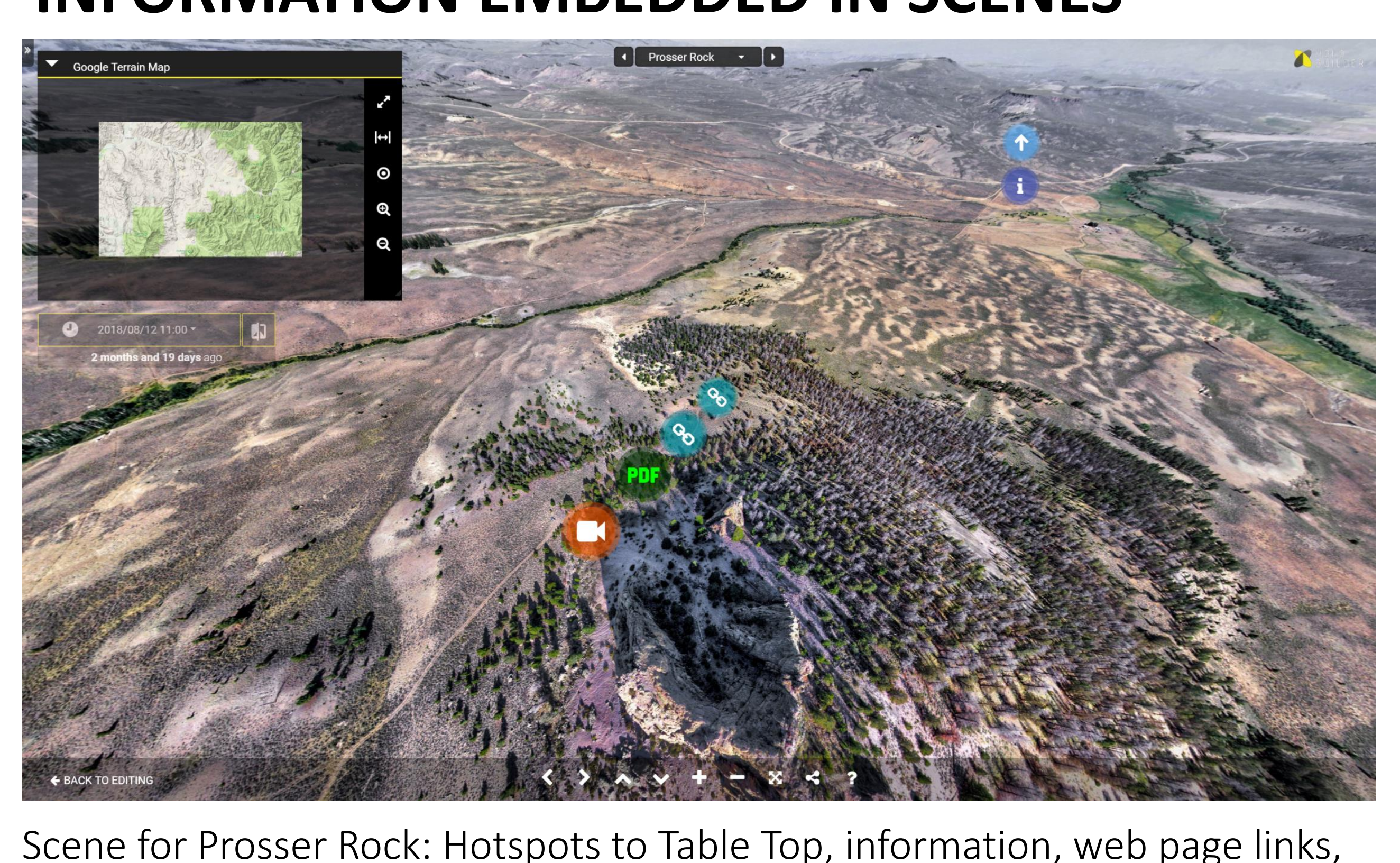

Scene for Prosser Rock: Hotspots to Table Top, information, web page links,
PDF documents, and videos are available in the scene. The 360-degree aerial panorama was created from pictures taken by a UAV.

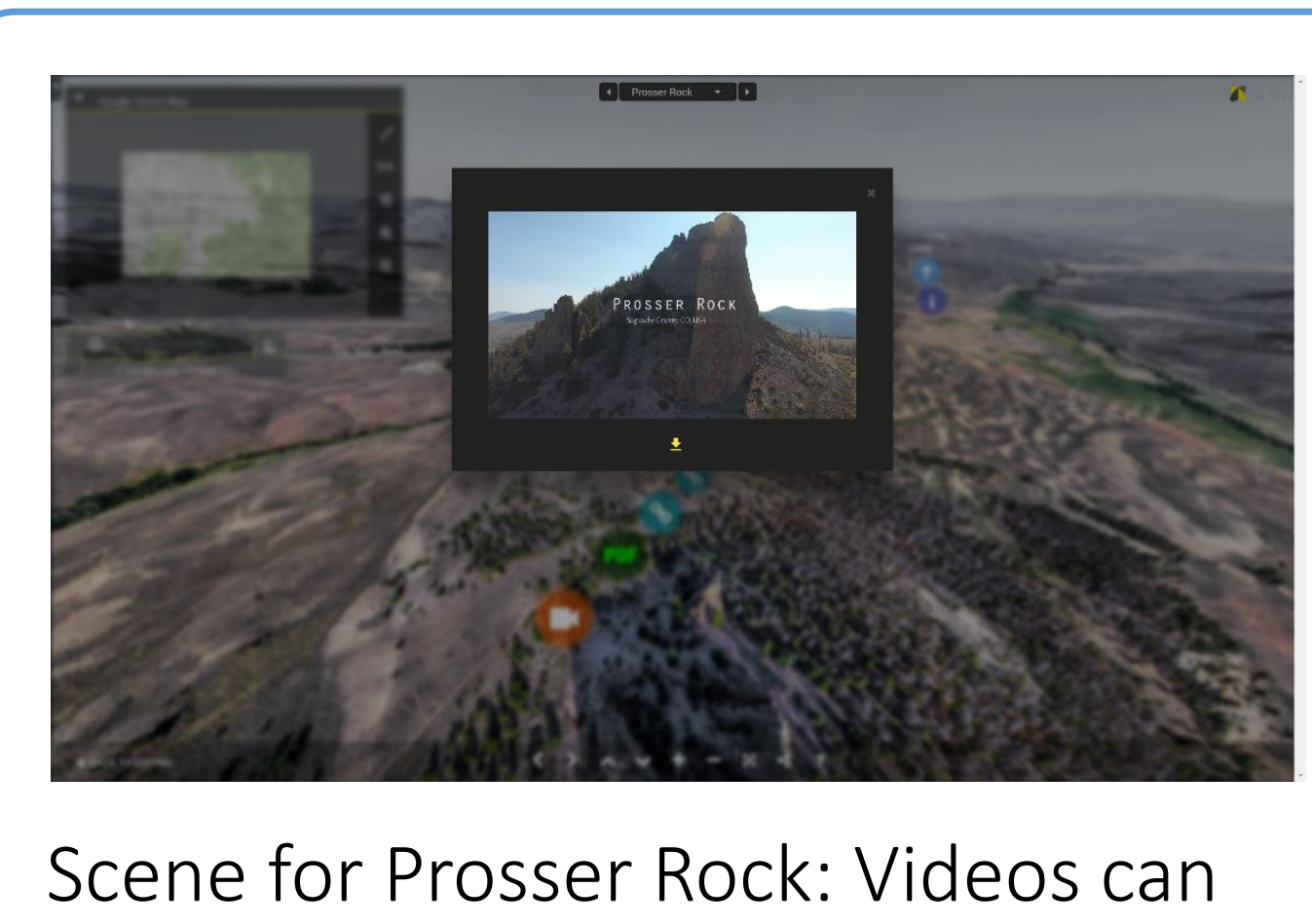
Scene for Prosser Rock:
be played inside a scene.

We have created AP360 for Prosser Rock, P2, and Table Top using pictures taken by an UAV, DJI Phantom 4

Lots of objects that link to the information, web links, PDF documents, and videos are available. 3D models were embedded in a scene as well, but they are not active objects which can be rotated or zoomed.

Terrain, topographic, and geologic maps were added to give students the geographical and geological understanding on the areas of interest.

3D MAP PLATFORM WITH GEOSPATIAL DATA AND 3D TILING CAPABILITY

We have created 3D map platform with 3D terrain models embedded as layers in the map environment. The 3D map environment is powered by CesiumJS Javascript engine. The 3D tiling capability of the platform allows quick loading of the area that is currently in the view.

We have used a UAV, DJI Phantom 4, to scan Table Top, Prosser Rock, and P2 in Saguache County and created their 3D models.

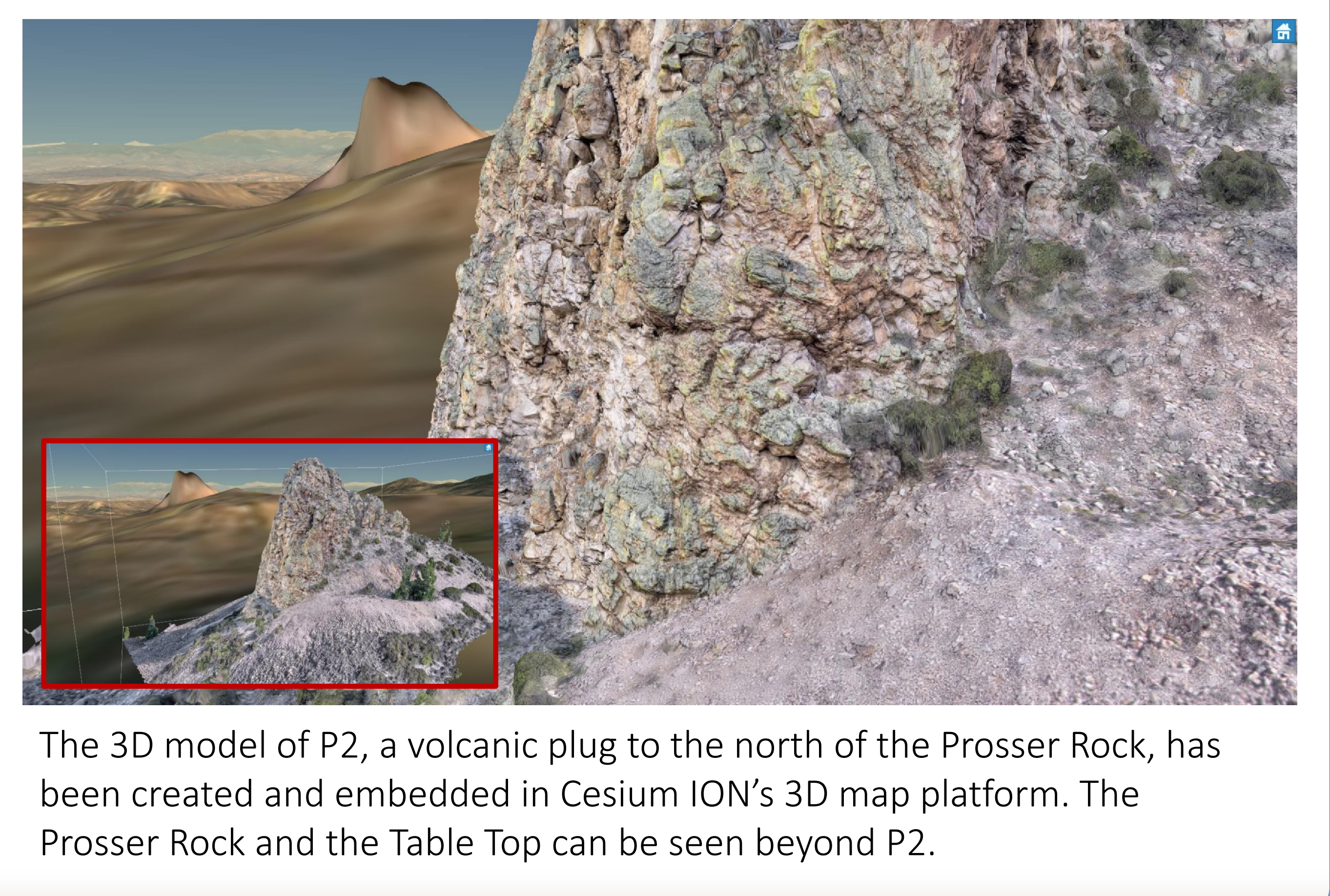

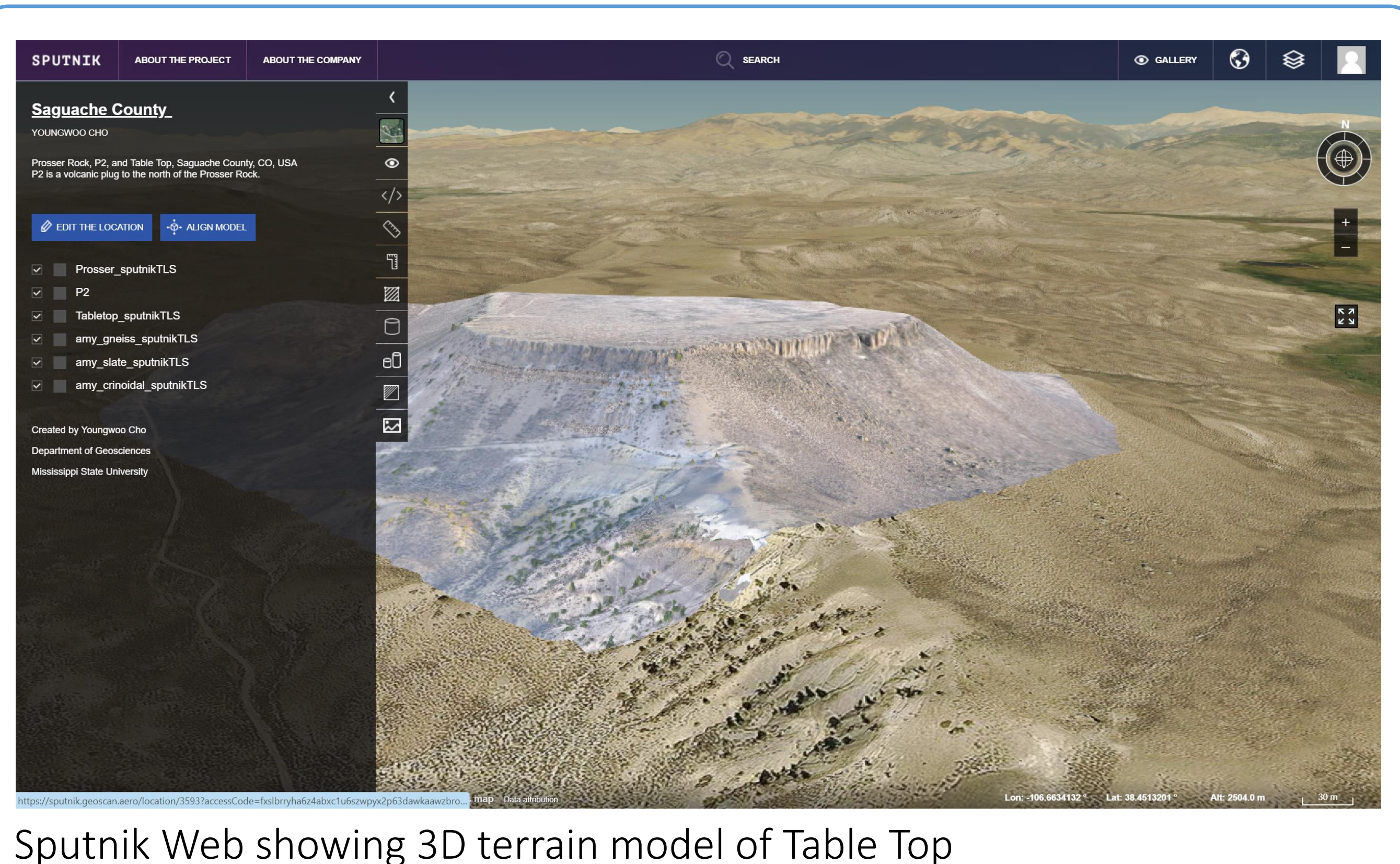

Geographical relationship can easily be understood with high definition 3D terrain models embedded on the 3D base map. The geospatial data help students measure the quantities such as length, height, distance, area, and volume of geospatial data.

\section{DISCUSSION \& CONCLUSION}

- 2D Tiled Gigapixel Images were very useful in investigating detailed texture and structures in road cuts along together with the entire view. However, lens focal length should be carefully chosen to provide a full details of the texture.

- 3D rock models proved to have sufficient resolution for visual inspection of specimens primarily due to the enhanced photographic techniques we have used.

- Virtual tour using 360-deg aerial panoramas is particularly good in overviewing the areas of interest. But, 3D models in HoloBuilder should be given a way that allows rotation and zoom.

- 3D map platforms were quite good to geospatially investigate the targets. Combined with some more coding, it will serve as the integrated environment for geologic mapping and visual inspection of areas of interest.

\section{ACKNOWLEDGMENT}

We want to acknowledge the support from the Department of Geosciences, Mississippi State University for high-performance computing and travel fund
to Colorado. We also acknowledge the travel grants for the GSA Indianapolis

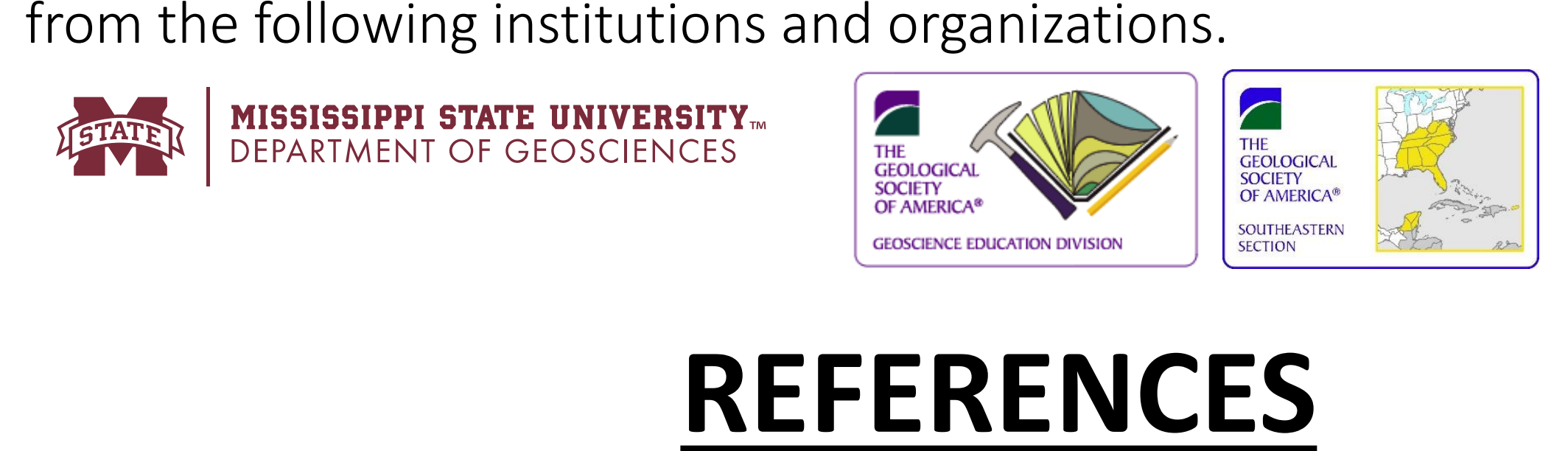

